\title{
Direct cost associated with acquired brain injury in Ontario
}

Amy Chen ${ }^{1}$, Ksenia Bushmeneva², Brandon Zagorski ${ }^{3}$, Angela Colantonio ${ }^{1,4}$, Daria Parsons ${ }^{1}$ and Walter P Wodchis ${ }^{1,2,3^{*}}$

\begin{abstract}
Background: Acquired Brain Injury (ABI) from traumatic and non traumatic causes is a leading cause of disability worldwide yet there is limited research summarizing the health system economic burden associated with ABI. The objective of this study was to determine the direct cost of publicly funded health care services from the initial hospitalization to three years post-injury for individuals with traumatic (TBI) and non-traumatic brain injury (nTBI) in Ontario Canada.

Methods: A population-based cohort of patients discharged from acute hospital with an ABI code in any diagnosis position in 2004 through 2007 in Ontario was identified from administrative data. Publicly funded health care utilization was obtained from several Ontario administrative healthcare databases. Patients were stratified according to traumatic and non-traumatic causes of brain injury and whether or not they were discharged to an inpatient rehabilitation center. Health system costs were calculated across a continuum of institutional and community settings for up to three years after initial discharge. The continuum of settings included acute care emergency departments inpatient rehabilitation (IR) complex continuing care home care services and physician visits. All costs were calculated retrospectively assuming the government payer's perspective.
\end{abstract}

Results: Direct medical costs in an ABI population are substantial with mean cost in the first year post-injury per TBI and $\mathrm{nTBI}$ patient being $\$ 32132$ and $\$ 38018$ respectively. Among both TBI and nTBI patients those discharged to IR had significantly higher treatment costs than those not discharged to IR across all institutional and community settings. This tendency remained during the entire three-year follow-up period. Annual medical costs of patients hospitalized with a brain injury in Ontario in the first follow-up year were approximately $\$ 120.7$ million for TBI and $\$ 368.7$ million for nTBI. Acute care cost accounted for $46-65 \%$ of the total treatment cost in the first year overwhelming all other cost components.

Conclusions: The main finding of this study is that direct medical costs in ABI population are substantial and vary considerably by the injury cause. Although most expenses occur in the first follow-up year ABI patients continue to use variety of medical services in the second and third year with emphasis shifting over time from acute care and inpatient rehabilitation towards homecare physician services and long-term institutional care. More research is needed to capture economic costs for ABI patients not admitted to acute care.

\footnotetext{
* Correspondence: walter.wodchis@utoronto.ca

${ }^{1}$ Toronto Rehabilitation Institute, Toronto, ON, Canada

${ }^{2}$ Institute of Health Policy Management and Evaluation, University of Toronto,

Toronto, ON, Canada

Full list of author information is available at the end of the article
}

\section{Biomed Central}

(c) 2012 Chen et al.; licensee BioMed Central Ltd. This is an Open Access article distributed under the terms of the Creative Commons Attribution License (http://creativecommons.org/licenses/by/2.0), which permits unrestricted use, distribution, and reproduction in any medium, provided the original work is properly cited. 


\section{Background}

Acquired Brain Injury (ABI) from both traumatic (such as falls and motor vehicle crashes) and non-traumatic (such as anoxia and brain tumors) causes is a leading cause of death and disability in North America [1,2]. An acquired brain injury is damage to the brain after birth and is not due to a congenital disorder developmental disability or a process which progressively damages the brain [3]. The National Center for Injury Prevention and Control estimated that $2 \%$ of US population live with disability as a result of brain injury from traumatic causes alone [4]. Annually 1.7 million people in the US sustain Traumatic Brain Injury (TBI) resulting in 275000 hospitalizations and 52000 deaths [5].

$\mathrm{ABI}$ patients have long-lasting impairments and their treatment requires significant health care resources [6-9]. Evidence suggests that utilization of medical and rehabilitation services by TBI patients remains high for several years post-discharge [10-13]. Cameron and colleagues [13] matched patients with TBI with a noninjured comparison group and found that the TBI cohort had more post-injury hospitalizations (rate ratio $(\mathrm{RR})=1.54 \quad 95 \% \quad \mathrm{CI}=1.39-1.71) \quad$ greater cumulative lengths of stay $(R R=5.1495 \% C I=3.29-8.02)$ and a greater post-injury physician claims rate $(R R=1.4495 \%$ $\mathrm{CI}=1.35-1.53)$ than the non-injured cohort. Advances in medical science in recent years may have resulted in better outcomes and higher survival probability for $\mathrm{ABI}$ patients with more patients directed to rehabilitation centers to augment their recovery [14]. However TBI patients admitted to the post-acute settings are medically complex have longer LOS and are at increased risk of re-hospitalization. The Canadian Institute of Health Information (CIHI) reported that the median length of stay (LOS) in rehabilitation centers for patients with brain dysfunction 36 days versus 21 days for average rehabilitation patient [15]. Patients with head injuries also had prolonged stays in other sub-acute facilities with the median LOS in complex continuing care of 92 days versus 40 days for all patients cared for in the same setting [16].

Despite the substantial financial burden of ABI there is limited research on the comprehensive costs associated with it. Significant attention has been devoted to estimating costs of TBI but this literature is subject to a number of limitations. In particular many studies examine only a subset of TBI patients such as patients with a specific level of injury severity $[17,18]$ occupation type [19] or a specific age [20]. Other findings are based on a small non-representative sample of patients [ $\left[\begin{array}{lll}10 & 11 & 20\end{array}\right]$ and the authors use the incidence rates to project sample-based cost estimates to the entire population. Some authors relied on patients' recall to identify their use of health services [10] or used systematic reviews of
TBI costing literature $[21,22]$. In addition most studies only covered costs related to the initial hospitalization [17,21-24]. Given that hospitalized TBI patients often face a prolonged recovery process and suffer from longlasting consequences of injury [9] such an approach clearly underestimates the true burden of TBI-related costs. Only a handful of studies reported costs across a continuum of care $[19,20]$ none of which were based on recent Canadian data. Past studies have focused exclusively on TBI and comparator heath system costs of non-traumatic brain injury (nTBI) have not been previously estimated.

The current study aims to address limitations of previous research. The present findings are based on recent and comprehensive data. The study examines health system burden in terms of direct costs to the public health care system for all ABI patients discharged from Ontario acute care hospitals between the years 2004-2008. Since Ontario is the most populous Canadian province representing $38 \%$ of Canadian population patient records analyzed in this study comprise a large and representative sample of the Canadian population of ABI patients which makes our results generalizable to the Canadian population. The findings can also be extended to other jurisdictions assuming that they share epidemiology and treatment patterns that are similar to the ones in Ontario. Ontario administrative data have been shown to provide reliable records of utilization of a variety of health care services based on data from provincial administrative healthcare databases [25-27]. In addition to estimating the medical costs of TBI we expand the previous studies by estimating direct costs for nTBI patients. The economic costs for TBI and nTBI are further broken down into those discharged to rehabilitation and those who were not. Rehabilitation is a costly service that is most often used for those who are most severely injured and suffer from more severe functional impairment. Therefore the results are presented this way to show costs for those with greater functional impairments. The primary objective of this study is to provide accurate and comprehensive estimates of direct medical costs from initial hospitalization up to 3 years postinjury among individuals with $\mathrm{ABI}$ for the years 2004-2008.

\section{Methods}

\section{Study population}

The study population consisted of all patients discharged alive from acute care with either traumatic or nontraumatic brain injury between April 12004 and March 31 2007. Patients in the cohort were followed retrospectively until the earlier of death or March 312008 and were stratified according to the cause of brain injury (TBI and nTBI) and their discharge disposition from an 
acute care hospital (inpatient rehabilitation or elsewhere). We identified 11970 cases of TBI and 31501 cases of nTBI using patients' associated ICD-10 codes in any diagnosis position in the Discharge Abstract Database (DAD) which records all hospital admissions in the province of Ontario and up to 25 diagnoses. Patients with the following diagnoses were classified into TBI category: fracture of skull intracranial injury late effects of injuries poisonings and toxic effects and other external causes. Patients with a stroke in any diagnosis position and patients with hospital-acquired $\mathrm{ABI}$ diagnosis were excluded. Diagnoses in nTBI group corresponded to the following ICD-10 codes: toxic effects of substances complications of surgical and medical care not elsewhere classified anoxia vascular insults brain tumours encephalitis metabolic encephalopathies and meningitis. The nTBI group did not include patients with a stroke as their most responsible diagnosis since this population represents a well-studied and unique impairment. The exact ICD-10 codes are reported in Table 1 . To ensure that we have identified new cases rather than readmissions patients who suffered from ABI in the year prior to their index injury were excluded from the analysis.

\section{Overview of cost calculation}

The focus for this study was the economic burden of patients with ABI in a publicly funded healthcare system. Both total and annualized per patient costs were calculated and reported. Annualized costs were estimated for all $A B I$ patients across a continuum of institutional and community care settings using a bottom-up costing approach. In particular treatment costs incurred in the follow-up period (up to three years after acute care admission) were calculated for acute care emergency department (ED) inpatient rehabilitation (IR) complex continuing care (CCC) home care services and physician visits. Since Ontario has universal public health care insurance program with supplementary workplace drug and disability insurance and private auto insurance all costs in this study were calculated from the government payer's perspective (Ontario Ministry of Health and Long-Term Care) and expressed in 2007 Canadian dollars. Cost calculation excluded any indirect and direct costs incurred by patient and family such as forgone income or associated out-of-pocket expenses or insurance compensation paid out by third-party payers.

Patient-specific health care utilization data were abstracted from provincial administrative databases and then linked across databases using patients' scrambled health card identifier. Data on acute hospital admission are stored in the Discharge Abstract Database (DAD) and the National Rehabilitation Reporting System (NRS) contains inpatient rehabilitation data. Data on utilization of homecare services and complex continuing care were obtained from the Home Care Reporting System (HCRS) and the Continuing Care Reporting System (CCRS) respectively. Although the quality of ABI diagnostic coding is insufficient to accurately identify $\mathrm{ABI}$ patients in the emergency departments all patients in the cohort were assumed to be admitted to ED prior to their hospitalization. Usage of physician services was identified from Ontario Health Insurance Plan (OHIP).

Unit costs of inpatient acute care ED visits home care and complex continuing care were obtained from the Ministry of Health and Long-Term Care (MOHLTC) Health Data Branch website [28] and are based on costing data submitted by service providers to the MOHLTC. The Health Data Branch provides financial advice to the MOHLTC ensures accountability of health care providers and develops funding allocations to support Ontario health system plans. Complex continuing care costs were derived from government-stipulated payment rates. Ambulatory costs were based on government-stipulated OHIP fee codes for specific service categories using the median reimbursement amount reported in 2007. Rehabilitation costs were based on unit case costs reported in the Joint Planning and Policy Committee's (JPPC) technical report [29].

Case cost calculation for acute care IR and CCC was based on a case-mix costing methodology in which each patient was assigned a weight representing the intensity of resource utilization during the stay. These patientspecific weights multiplied by a provincial unit cost for a given care setting provide an estimate of the case cost. In acute care and IR settings the unit cost reported the MOHLTC is per weighted case (CPWC) where as in $\mathrm{CCC}$ the unit cost is per weighted day. Unit costs for ED home care and physician visits measure the average cost per visit or per hour. Total cost for these services is calculated by multiplying the number of visits or hours of service that the patient utilized by the provincial-average cost.

Total costs were calculated by aggregating individual costs over one, two and three year periods for each type of health care service. Average per patient costs were estimated as unconditional mean by dividing the total costs by the total number of ABI patients in each subgroup at the start of each study period.

\section{$E D$ visits and acute care}

Emergency department visits were valued at \$187 (CAD 2007) which was the average (non-weighted) cost of an ED visit reported by the MOHLTC Health Data Branch [28]. Due to data availability assumptions were required to measure ED utilization by ABI patients. These assumptions were three-fold: 1) all TBI and nTBI patients in the cohort required an ED visit prior to index 
Table 1 ICD Codes for Conditions leading to Acquired Brain Injury ( $\mathrm{ABI}$ )Final List of Codes based on Feedback and Studies -Ontario Neurotrauma Foundation ABI Pilot Project

\section{Traumatic Causes}

Diagnosis

ICD-10 Code and

Description

1. Fracture of the skull

2. Intracranial Injury excluding those with skull fracture

3. Other causes including late effects of injuries poisonings toxic effects \& other external causes involving skull and facial

\section{S02.0 Skull \\ S02.1 Base of skull}

S02.9 Skull and facial bones 502.7 Multiple fractures bones

S02.89 Fractures of other unspecified skull and facial bones

S06.0 Concussion

S06.2 Diffuse brain injury

S06.4 - Epidural Hemorrhage

S06.5 - Traumatic subdural

hemorrhage

S06.6 - Traumatic subarachnoid hemorrhage

S06.8 - Other intracranial injuries

S06.1 - Traumatic cerebral edema

S06.3 - Focal brain injury

S06.9 - Intracranial injury unspecified

S09.7 - Multiple injuries of head

S09.8 - Other unspecified injuries of head

S09.9 - Unspecified injury of head

T90.2 - Sequelae of fracture of skull and facial bones

T90.5 - Sequelae of intracranial injury

T90.8 - Sequelae of other specified injuries of head

T90.9 - Sequelae of unspecified injury of head

T96 - Sequelae of poisoning by drugs medicaments and biological substances

T97 - Sequelae of toxic effects of substances chiefly nonmedicinal as to source

T98.2 - Sequelae of certain early complications of trauma

Table 1 ICD Codes for Conditions leading to Acquired Brain Injury ( $\mathrm{ABI}$ )Final List of Codes based on Feedback and Studies -Ontario Neurotrauma Foundation ABI Pilot Project (Continued)

\section{Non Traumatic Causes}

\begin{tabular}{ll}
\hline Diagnosis & $\begin{array}{l}\text { ICD-10 Code and } \\
\text { Description }\end{array}$ \\
\hline $\begin{array}{ll}\text { 1. Toxic effects of substances } \\
\text { chiefly non-medical as to }\end{array}$ & T51- Toxic effect of alcohol \\
source & T56- Toxic effect of metals \\
& $\begin{array}{l}\text { T58- Toxic effect of carbon } \\
\text { monoxide }\end{array}$
\end{tabular}

2. Complications of surgical \& medical care not elsewhere classified

3. Anoxia

4. Vascular insults

(Aneurysm and vascular malformations)

RVL Needs clarification

Aneurysm 177.0

Malformation Q28.0 -Q28.9

Traumatic aneurysm T14.5

5. Brain Tumours
G93.8 Other specified disorders of the brain (including postradiation encephalopathy)

G93.1- Anoxic brain damage (includes all causes of anoxia except those occurring following abortions ectopic pregnancy labour \& delivery \& newborn)

T75.1- Drowning and nonfatal submersion

T71- Asphyxiation suffocation (by strangulation)

162.0 - Subdural hemorrhage

162.9 - Unspecified intracranial hemorrhage

C71 - Malignant neoplasm of brain

C79.3 - Secondary malignant neoplasm of brain and cerebral meninges

D33.0-D33.3 - Benign neoplasm of brain and other parts of central nervous system

D32.0 - Benign neoplasm of cerebral meninges

D43 - Neoplasm of uncertain or unknown behaviour of brain and central nervous system

C70 - Malignant neoplasm of brain

D43.2 - Neoplasm of brain unspecified

D42.0 - Neoplasm of uncertain or unknown behaviour of cerebral meninges 
Table 1 ICD Codes for Conditions leading to Acquired Brain Injury (ABI)Final List of Codes based on Feedback and Studies -Ontario Neurotrauma Foundation ABI Pilot Project (Continued)

6. Encephalitis

7. Metabolic Encephalopathies

8. Meningitis

A81.1- Subacute sclerosing encephalitis

B00.4 - Herpesviral meningoencephalitis

B05.0 - Postmeasles encephalitis

A83.0 - Japanese encephalitis

A83.2 - Eastern equine encephalitis

A86.0 - Unspecified viral encephalitis

G04.0 - Acute Disseminated encephalitis

G04.8 - Other encephalitis myelitis and encephalomyelitis

G04.9 - Encephalitis myelitis and encephalomyelitis unspecified

B01.1 - Varicella encephalitis

B02.0 - Zoster encephalitis

G05. - Encephalitis myelitis and encephalomyelitis in diseases classified elsewhere

E10.0 (Type I) E11.0 (Type II)

E13.0 - Other specified diabetes mellitus with coma

E14.0 - Unspecified diabetes mellitus with coma

E15 - Nondiabetic hypoglycaemic coma

F07.2 - post concussion syndrome

G06.0 - intracranial abscess and granuloma

G06.1 - Intraspinal abscess and granuloma G06.2 - Extradural and subdural abscess unspecified

G93.0 - Cerebral cysts

A87 - Viral meningitis

B01.0 - Varicella meningitis

B37.5 - Candidal meningitis

G00 - Bacterial meningitis not elsewhere classified

G01* - Meningitis in bacterial diseases classified elsewhere

G02* - Meningitis in other infectious and parasitic diseases classified elsewhere

G03. - Meningitis due to other and unspecified causes hospitalization 2) a single ED visit per patient was assumed and additional ED visits throughout a year were not included and 3) mild ABI cases that were discharged home following an ED visit and those that were seen in physician offices only or went undetected were not included in the present study and thus their ED visit costs were unaccounted for. The first assumption is likely to be satisfied for TBI patients but may overestimate ED visits for nTBI patients since some of the nTBI patients could be admitted to hospital for scheduled procedures. Because TBI is of an accidental nature the majority of TBI patients have non-scheduled hospitalizations and thus require an ED visit. Ontario Neurotrauma Foundation (ONF) reported that for TBI patients falls, being struck by and motor vehicle crashes together account for $78.5 \%$ of all TBI acute care admissions [30].

The second and third assumptions underestimate total ED costs. The second assumption is not very restrictive since approximately $92 \%$ of $\mathrm{ABI}$ patients have a single ED episode and only minority of patients have multiple ABI-related ED visits [30]. Also based on our cohort definition the present study omits patients with mild $A B I$ who were treated in the ED but were not admitted to acute care. Among TBI patients there may be many mild cases. Data by ONF suggest that in 2006 there were approximately fifteen thousand TBI patients admitted to ED but only five thousand were treated in acute care [30]. Given the above two assumptions our estimates of ED costs are conservative and provide a lower boundary for ABI-related ED hospitalization costs. Case cost calculation for patients in acute hospitals was based on casemix methodology described earlier. Hospital patients are categorized into homogeneous case mix groups (CMG) that takes account of patients' clinical condition or medical procedures. Patient CMG age co-morbidities specific interventions and the length of hospital stay determine his/her resource intensity weight (RIW) that approximates the amount of hospital resources used up during the hospitalization relative to the average inpatient $(\mathrm{RIW}=1.0)$. Following this each case cost is estimated by multiplying the weight by provincial cost per weighted case for acute care valued at \$5212 in 2007 [28].

\section{Complex continuing care}

Each continuing care resident is classified into one of 44 Resource Utilization Groups (RUG-III) according to clinical condition physical functioning and treatment in the last 14 days since admission. Each RUG-III group has an associated Case Mix Index (CMI) (functionally similar to acute care RIWs) that approximates the daily amount of medical resources used to care for residents in that group relative to the average resident. To calculate total CCC case costs we multiplied each patient's 
length of stay by the corresponding CMI to measure weighted patient days and then multiplied by the provincial average cost per weighted patient day ( $\$ 469.2$ in 2007).

\section{Inpatient rehabilitation}

The approach used to calculate rehabilitation-related case costs was similar to the case mix methodology used to calculate acute and CCC costs. Each patient entering the IR facility is assigned to one of the Rehabilitation Patient Groups (RPG) based on the motor and cognitive Functional Independence Measure (FIM) scores age and rehabilitation client code. Patients in each of the RPGs are then given Rehabilitation Cost Weights (RCW) which approximate their resource intensity relative to the average rehabilitation patient. Individual rehabilitation case cost is calculated by multiplying each patient's weight by the rehabilitation cost per weighted case (CPWC). Unlike acute CPWC rehabilitation CPWC is not reported every year. The rehabilitation CPWC used in this study was based on costs reported by JPPC for the 2004/05 fiscal year and then extrapolated to 2007 using the relative changes in acute hospital case costs [29].

\section{Home care}

Total costs for home care services were estimated using patient's total annual number of visits or hours in each of 14 service categories multiplied by the average provincial costs for each service reported by the MOHLTC. Per patient average annual cost was estimated by dividing total costs by the number of patients in each subgroup.

\section{Ambulatory care}

Although this has recently begun to change traditionally primary care physicians in Canada have been paid according to a fee-for-service model [31,32]. Fee-for-service physician costs were obtained based on the total number of visits by service type and the median reimbursed amount for the associated fee codes in 2007 fiscal year. Annualized per patient costs were calculated by dividing the cumulative cost by the total number of patients in each subgroup.

\section{Privacy and ethics}

This study received ethics approval from the Toronto Rehabilitation Institute Research Ethics Board. All investigators and staff involved in the study signed confidentiality agreements and analyses were conducted with de-identified data.

\section{Results}

The characteristics of the TBI and nTBI cohorts can be found in Tables 2 and 3. Overall 10\% of the TBI cohort and $9 \%$ of the nTBI cohort were discharged to rehabilitation. The sex distribution was similar for both discharge destinations in each of the cohorts. In both cohorts older patients were more likely to be discharged to rehabilitation compared to younger patients. In addition those discharged to rehab were more likely to live in non-rural areas and have longer inpatient lengths of stay. Among the TBI cohort those discharged to rehabilitation were more likely to have higher Charlson Comorbidity Index scores and to have been caused by a

Table 2 Demographic and characteristics of TBI Cohort

\begin{tabular}{|c|c|c|c|}
\hline & $\begin{array}{l}\text { Discharged } \\
\text { to Rehab } \\
\text { [N (\%)] }\end{array}$ & $\begin{array}{l}\text { Not discharged } \\
\text { to Rehab } \\
\text { [N (\%)] }\end{array}$ & $\begin{array}{l}\text { Total } \\
\text { [N (\%)] }\end{array}$ \\
\hline \multicolumn{4}{|l|}{ Sex } \\
\hline Female & $403(34)$ & $3717(34)$ & $4120(34)$ \\
\hline Male & $784(66)$ & 7066 (66) & $7850(66)$ \\
\hline \multicolumn{4}{|l|}{ Age } \\
\hline$<18$ & $21(2)$ & $2460(23)$ & $2481(21)$ \\
\hline $18-34$ & $262(22)$ & $1891(18)$ & $2153(18)$ \\
\hline $35-54$ & $306(26)$ & $2114(20)$ & $2420(20)$ \\
\hline $55-64$ & $144(12)$ & $1040(10)$ & $1184(10)$ \\
\hline $65-74$ & $133(11)$ & $1061(10)$ & $1194(10)$ \\
\hline $75+$ & $321(27)$ & $2217(21)$ & $2538(21)$ \\
\hline \multicolumn{4}{|l|}{ Residence } \\
\hline Non-Rural & $1016(86)$ & $8570(80)$ & $9586(80)$ \\
\hline Rural & $171(14)$ & $2213(20)$ & $2384(20)$ \\
\hline \multicolumn{4}{|c|}{ Charlson Comorbidity Index } \\
\hline $0-1$ & $1035(87)$ & $10018(93)$ & $11053(92)$ \\
\hline $2-3$ & $132(11)$ & $615(6)$ & $747(6)$ \\
\hline $4+$ & $20(2)$ & $150(1)$ & $170(1)$ \\
\hline \multicolumn{4}{|c|}{ Inpatient LOS (days) } \\
\hline $1-2$ & $5(0)$ & $2760(25)$ & $2765(23)$ \\
\hline $3-5$ & $56(5)$ & $2099(29)$ & $3155(26)$ \\
\hline $6-11$ & $224(19)$ & $2451(23)$ & $2675(22)$ \\
\hline $12+$ & $902(76)$ & $2473(23)$ & $3375(28)$ \\
\hline \multicolumn{4}{|c|}{ Motor Vehicle Related } \\
\hline Yes & $416(35)$ & $2099(20)$ & $2515(21)$ \\
\hline No & $771(65)$ & $8684(80)$ & 9455 (79) \\
\hline \multicolumn{4}{|l|}{ TBI category* } \\
\hline Fracture of Skull & $440(37)$ & $3257(30)$ & 3697 (30) \\
\hline Intercranial Injury & $1098(92)$ & $8916(82)$ & 10014 (83) \\
\hline Other causes & $30(2)$ & $670(6)$ & $700(5)$ \\
\hline
\end{tabular}

* Categories are not mutually exclusive $32 \%$ of patients have more than one listed diagnosis. 
Table 3 Demographic and characteristics of nTBI Cohort $\begin{array}{lll}\begin{array}{l}\text { Discharged } \\ \text { to Rehab }\end{array} & \begin{array}{l}\text { Not discharged } \\ \text { to Rehab }\end{array} & \text { Total } \\ \text { [N (\%)] }\end{array}$ [N (\%)] [N (\%)]

\begin{tabular}{|c|c|c|c|}
\hline \multicolumn{4}{|l|}{ Sex } \\
\hline Female & $1308(47)$ & $13741(48)$ & $15049(48)$ \\
\hline Male & $1496(53)$ & $14956(52)$ & $16452(52)$ \\
\hline \multicolumn{4}{|l|}{ Age } \\
\hline$<18$ & $10(0)$ & $2450(9)$ & $2460(8)$ \\
\hline $18-34$ & $106(4)$ & $2235(8)$ & $2341(7)$ \\
\hline $35-54$ & $485(17)$ & $6056(21)$ & $6541(21)$ \\
\hline $55-64$ & $450(16)$ & $4519(16)$ & $4969(16)$ \\
\hline $65-74$ & $644(23)$ & $5298(18)$ & $5942(19)$ \\
\hline $75+$ & $1109(40)$ & $8129(28)$ & $9248(29)$ \\
\hline \multicolumn{4}{|l|}{ Residence } \\
\hline Non-Rural & $2444(87)$ & $23856(83)$ & $26300(84)$ \\
\hline Rural & $360(13)$ & $4841(17)$ & $5201(16)$ \\
\hline \multicolumn{4}{|l|}{ Charlson Comorbidity Index } \\
\hline $0-1$ & $1445(52)$ & $14675(51)$ & $16120(51)$ \\
\hline $2-3$ & $904(32)$ & $6885(24)$ & $7789(25)$ \\
\hline $4+$ & $455(16)$ & $7137(25)$ & $7582(24)$ \\
\hline \multicolumn{4}{|l|}{ Inpatient LOS (days) } \\
\hline $1-2$ & $24(1)$ & $2911(10)$ & $2935(9)$ \\
\hline $3-5$ & $168(6)$ & $6943(24)$ & $7111(23)$ \\
\hline $6-11$ & $584(21)$ & $8148(28)$ & $8732(28)$ \\
\hline $12+$ & $2028(72)$ & $10695(37)$ & $12723(40)$ \\
\hline \multicolumn{4}{|l|}{ Type of nTB|* } \\
\hline Brain Infection & $274(10)$ & $4023(14)$ & $4297(14)$ \\
\hline Brain Tumour & $1888(67)$ & $17172(60)$ & $19060(60)$ \\
\hline $\begin{array}{l}\text { Metabolic } \\
\text { encephalopathies }\end{array}$ & 109 (3) & $2086(7)$ & $2195(7)$ \\
\hline Anoxia & $121(4)$ & $902(3)$ & $1023(3)$ \\
\hline $\begin{array}{l}\text { Complications of Surgical } \\
\text { and Medical Care }\end{array}$ & $74(3)$ & $754(3)$ & $828(3)$ \\
\hline Vascular Insults & $358(13)$ & $1065(6)$ & $2423(8)$ \\
\hline Toxic Effects & $12(0)$ & $1918(7)$ & $1930(6)$ \\
\hline
\end{tabular}

* Categories are not mutually exclusive $0.9 \%$ of patients have more than one diagnosis.

motor vehicle collision. Among the nTBI cohort the majority of patients had a brain tumor as a diagnosis $(60 \%)$ among those discharged to rehabilitation; there was a higher percentage that had brain tumour (67\%) and vascular insults (13\%) as a diagnosis. The identified diagnostic categories for TBI patients were broader and more heterogeneous. Just over $32 \%$ of patients were represented in more than one diagnosis category. nTBI patient diagnostic categories were more distinct with only $0.9 \%$ represented in more than one diagnosis category.
A detailed breakdown of the average direct per-patient costs appears in Table 4. Several important features of the cost structure emerge from the table. Treatment of nTBI patients and of patients discharged to rehabilitation facility is more expensive relative to TBI patients and patients not discharged for rehabilitation respectively in the first year and in subsequent years. Although costs decrease with time since the injury they remain substantial three years after the index hospitalization. The estimated average direct medical cost in the first year following an acute care admission for TBI and nTBI patients was $\$ 32132$ and $\$ 38018$ respectively. ABI patients who required rehabilitation had considerably higher costs than those who were discharged from acute to other destinations; a result that holds for both traumatic (\$93340 versus \$25394) and non-traumatic injuries (\$82241 versus \$33697). This was not only due to the fact that these patients utilized more rehabilitation services but also because they had higher overall treatment costs for physician home care and other health care services.

In the first year post-injury the initial hospital stay accounted for the largest cost in all sub-groups of patients. For TBI patients discharged to IR cost associated with acute care stay made up $46 \%$ of the total per-patient cost followed by rehabilitation (36\%) and CCC (9\%). Acute care cost for TBI patients not discharged to inpatient rehabilitation although smaller in magnitude was the largest contributor to total cost (65\%). CCC accounted for $12 \%$ of the total per-patient cost among those not discharged to inpatient rehabilitation. The distribution of costs was very similar for TBI and nTBI patients once the discharge destination was taken into account (Figure 1).

The mean annualized treatment costs decreased dramatically in second year post injury with expenditure per TBI and nTBI patient being $\$ 2580$ and $\$ 4698$ respectively. Ongoing homecare physician and CCC care accounted for proportionally more costs in the second and third year. Ninety-one percent of TBI patients and $88 \%$ of nTBI patients (data not shown) utilized physician services in the second year which accounted for 36\% and $68 \%$ of the respective total mean cost. Only $4.5 \%$ and $1.8 \%$ of TBI and nTBI patients were readmitted to acute care in the second year. There was a very slight decrease in the total average costs in the third year relative to the second for both TBI and nTBI patients with per patient cost remaining above two- and four thousand dollars respectively.

\section{Discussion}

Canada is among other developed countries that are currently facing the challenge of controlling their publicly funded health care expenses and mounting fiscal debt. 
Table 4 Mean direct costs per ABI patient across health care settings in the first 3 years since injury

\begin{tabular}{|c|c|c|c|c|c|c|}
\hline & & TBI & & & nTBI & \\
\hline & All TBI Pts. & DC to IR & Not DC to IR & All nTBI Pts. & DC to IR & Not DC to IR \\
\hline Year $1[\mathrm{~N}]$ & [11970] & [1187] & [10783] & {$[31501]$} & {$[2804]$} & {$[28697]$} \\
\hline & Mean Annua & (standard d & & Mean Annual & standard de & \\
\hline Emergency Department & $\$ 187$ & $\$ 187$ & $\$ 187$ & $\$ 187$ & $\$ 187$ & $\$ 187$ \\
\hline Acute Care & $\$ 19083$ & $\$ 43123$ & $\$ 16436$ & $\$ 23078$ & $\$ 37953$ & $\$ 21625$ \\
\hline & (72495) & $(43441)$ & (74538) & $(77157)$ & $(45451)$ & $(79431)$ \\
\hline Inpatient Rehabilitation & $\$ 5363$ & $\$ 33646$ & $\$ 2250$ & $\$ 3149$ & $\$ 23610$ & $\$ 1150$ \\
\hline & $(22191)$ & $(43062)$ & (15649) & (13379) & $(29211)$ & $(8261)$ \\
\hline Complex Continuing Care & $\$ 3535$ & $\$ 8168$ & $\$ 3025$ & $\$ 5626$ & $\$ 11855$ & $\$ 5017$ \\
\hline & (28259) & $(40179)$ & $(26576)$ & (32105) & (49895) & (29734) \\
\hline Homecare & $\$ 722$ & $\$ 993$ & $\$ 692$ & $\$ 1626$ & $\$ 2104$ & $\$ 1580$ \\
\hline & (2259) & $(2439)$ & $(2236)$ & (3856) & (3793) & (3859) \\
\hline Physician & $\$ 3242$ & $\$ 7223$ & $\$ 2804$ & $\$ 4352$ & $\$ 6532$ & $\$ 4138$ \\
\hline & $(5122)$ & $(8152)$ & $(4458)$ & $(4251)$ & $(4702)$ & $(4143)$ \\
\hline Annual Cost & $\$ 32132$ & $\$ 93340$ & $\$ 25394$ & $\$ 38018$ & $\$ 82241$ & $\$ 33697$ \\
\hline Year $2[\mathrm{~N}]$ & [11294] & [1107] & [10187] & [23049] & [2372] & {$[20677]$} \\
\hline Emergency Department & $\$ 3$ & $\$ 5$ & $\$ 3$ & $\$ 9$ & $\$ 8$ & $\$ 9$ \\
\hline Acute Care & $\$ 377$ & $\$ 462$ & $\$ 368$ & $\$ 787$ & $\$ 689$ & $\$ 798$ \\
\hline & (5434) & (3894) & (5573) & $(6027)$ & $(5855)$ & $(6047)$ \\
\hline Inpatient Rehabilitation & $\$ 148$ & $\$ 464$ & $\$ 114$ & $\$ 127$ & $\$ 381$ & $\$ 98$ \\
\hline & $(3720)$ & $(5776)$ & (3428) & $(2545)$ & $(4664)$ & $(2177)$ \\
\hline Complex Continuing Care & $\$ 606$ & $\$ 1219$ & $\$ 540$ & $\$ 1018$ & $\$ 2067$ & $\$ 898$ \\
\hline & $(11297)$ & (18599) & (10192) & (13093) & (19010) & $(12228)$ \\
\hline Homecare & $\$ 512$ & $\$ 892$ & $\$ 471$ & $\$ 1242$ & $\$ 1934$ & $\$ 1162$ \\
\hline & $(2436)$ & (3705) & $(2251)$ & $(4146)$ & $(5068)$ & $(4019)$ \\
\hline Physician & $\$ 934$ & $\$ 1325$ & $\$ 892$ & $\$ 1515$ & $\$ 1790$ & $\$ 1483$ \\
\hline & $(1607)$ & $(1600)$ & $(1602)$ & $(2226)$ & (3076) & $(2104)$ \\
\hline Annual Cost & $\$ 2580$ & $\$ 4367$ & $\$ 2388$ & $\$ 4698$ & $\$ 6869$ & $\$ 4448$ \\
\hline Year $3[\mathrm{~N}]$ & [9068] & [897] & [8171] & [17325] & [1799] & [15526] \\
\hline Emergency Department & $\$ 1$ & $\$ 1$ & $\$ 1$ & $\$ 4$ & $\$ 4$ & $\$ 4$ \\
\hline Acute Care & $\$ 116$ & $\$ 287$ & $\$ 98$ & $\$ 263$ & $\$ 350$ & $\$ 254$ \\
\hline & $(2542)$ & $(4935)$ & $(2154)$ & (2915) & (3482) & $(2845)$ \\
\hline Inpatient Rehabilitation & $\$ 0$ & $\$ 0$ & $\$ 0$ & $\$ 83$ & $\$ 169$ & $\$ 74$ \\
\hline & & & & $(2056)$ & $(2694)$ & $(1971)$ \\
\hline Complex Continuing Care & $\$ 483$ & $\$ 344$ & $\$ 498$ & $\$ 1045$ & $\$ 2120$ & $\$ 922$ \\
\hline & (6698) & $(3675)$ & (6944) & (12303) & $(15052)$ & (11954) \\
\hline Homecare & $\$ 606$ & $\$ 1086$ & $\$ 555$ & $\$ 1378$ & $\$ 2305$ & $\$ 1271$ \\
\hline & $(3695)$ & $(4633)$ & (3573) & (5596) & (6520) & (5469) \\
\hline Physician & $\$ 1028$ & $\$ 1433$ & $\$ 984$ & $\$ 1625$ & $\$ 1901$ & $\$ 1593$ \\
\hline & (2315) & (5188) & (1744) & $(2720)$ & $(2751)$ & $(2715)$ \\
\hline Annual Cost & $\$ 2234$ & $\$ 3151$ & $\$ 2136$ & $\$ 4398$ & $\$ 4544$ & $\$ 2847$ \\
\hline
\end{tabular}



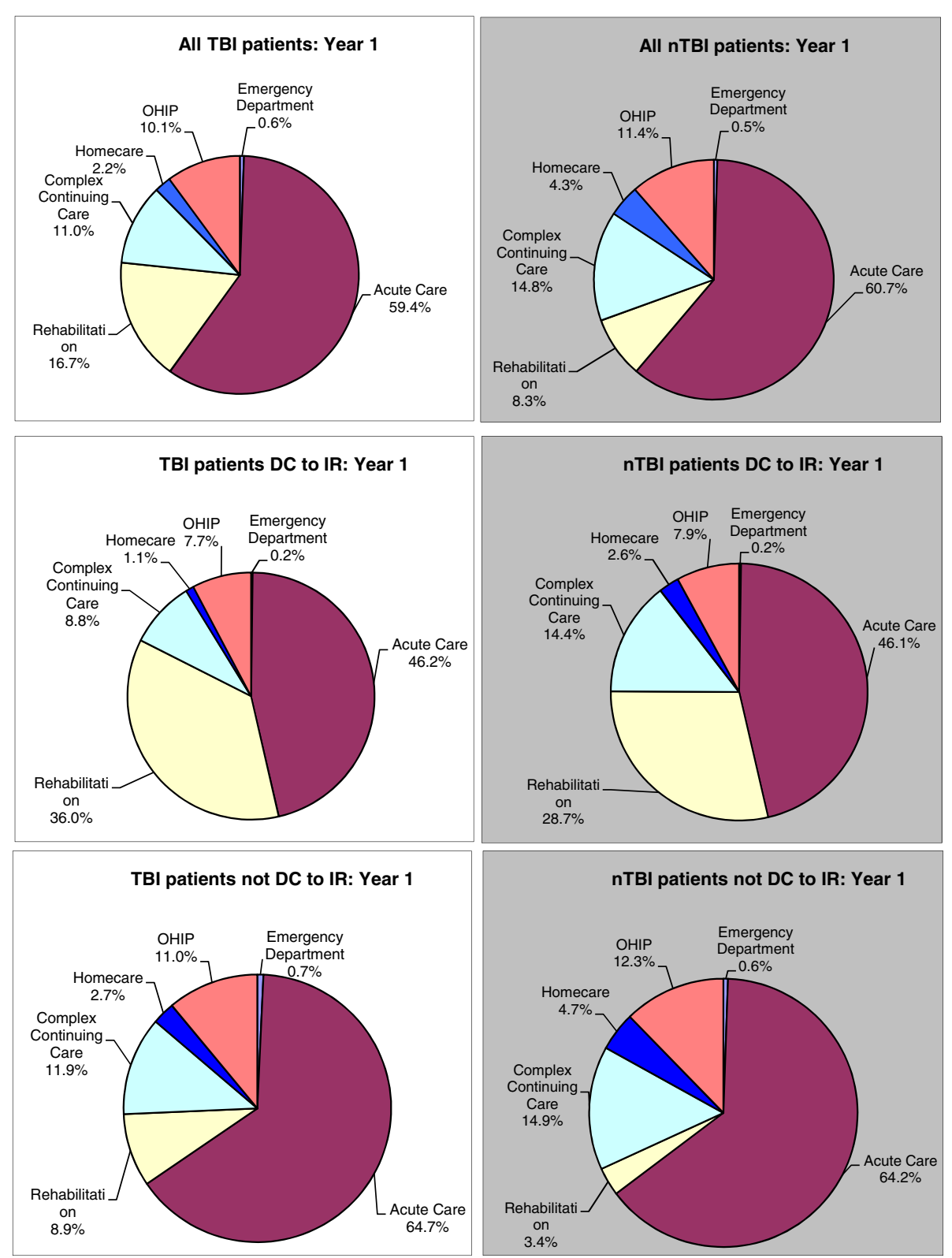

Figure 1 Cost distribution across health care system in the first year since discharge (DC).

In 2008 health care expenditure totalled CAD \$141.4 billion making up $10.7 \%$ of Canadian GDP and it is projected to continue to increase in the future [33]. In light of these challenges policy- and decision-makers have turned their attention to cost-of-illness (COI) studies to help direct attention to areas for improving health care accountability and long-term sustainability of health care expenditures $[34,35]$. The present study highlights considerable individual and total system costs for the treatment of ABI patients in Canada.

This population-based study followed a cohort of ABI patients discharged from acute care in 2004 - 2008 and examined the direct cost of publicly financed health care services in the first 3 years since the brain injury. It presented comprehensive information on direct costs of ABI by including costs across the entire health care system such as ED and acute inpatient costs, the costs of rehabilitation following the index event as well as CCC home care and physician costs. It also uses recent Canadian data and is the first costing study of ABI conducted on such wide scale and scope. Our main findings are four-fold: 1) treatment of nTBI patients is more expensive than of TBI patients both per patient and on aggregate; 2) discharge to inpatient rehabilitation is associated with higher treatment costs; 3) health care costs decrease over time but remain significant even 
after 3 years; and 4) for all subgroups of patients the highest cost in the first year was acute care while the highest three costs in the second and third year were physician and home care services and CCC.

Mean cost of treatment for an nTBI patient was only $18 \%$ higher than that for a TBI patient in the first postinjury year; however in the second and third follow-up years nTBI costs were nearly double those for TBI (Table 2). This suggests that on average nTBI patients suffer longer lasting health impairments and require substantially more intensive ongoing care than patients with TBI. The costs in the first year post-injury for TBI patients are mainly driven by intensive utilization of acute care rehabilitation and CCC services which corroborates with findings reported by Canadian Institute of Health Information (CIHI) $[10,11]$. CIHI estimated that that the median LOS in rehabilitation and CCC facilities for patients with head injury was more than two times that of an average patient. Earlier analyses of a similar population also showed that intensity of acute care (length of stay special care days) were positively associated with inpatient rehabilitation. [36] For patients with nTBI first-year costs were largely influenced by the cost of acute care CCC and physician visits.

Patients discharged to inpatient rehabilitation were more costly that those who were not. These findings are consistent with other studies that linked injury severity to higher treatment costs [11 2023 36]. Brooks et al. [20] grouped patients by severity in two ways: based on the Abbreviated Injury Scale (AIS) and by patient's rehabilitation discharge status. Brooks and colleagues found that costs increased with injury severity for both severity metrics. Vangel et al. [11] found that motor deficiency was a significant predictor of higher future medical billings. The study by Brooks et al. reported that the first year cost including initial hospitalization and follow-up services was \$151149 (\$228626 in 2007 dollars) per TBI patient discharged to IR and $\$ 16813$ (\$25431 in 2007 dollars) per TBI patient not discharged to IR. Our cost estimate for TBI patients not discharged to IR is slightly above the one reported by Brooks et al. potentially in part because we capture costs for patients admitted to IR at a later point in the year while Brooks et al. did not allow for this. On the other hand our cost per TBI patient discharged to IR is significantly below Brooks'. The latter disparity may be due to the fact that the study by Brooks et al. was based on US data and potentially reflects the difference in health care costs and provision between Canada and the US; in particular the use of technologies and rehabilitation services may be relatively more intensive and expensive in the US. Overall the cost of treatment of patients with $A B I$ in the first year post-injury is significant and comparable in magnitude to other health care conditions such as cardiac arrhythmia (\$22000), stroke (\$34000) and hip fracture (\$35000) [37]. A report to the Ontario Neurotrauma Foundation estimated approximately five thousand TBI and eleven thousand nTBI related hospitalizations in Ontario per year [30]. Total medical costs for TBI and nTBI patients in Ontario in the first follow-up year are approximately $\$ 120.7$ and $\$ 368.7$ million respectively with acute care cost overwhelming all other cost components. Assuming that incidence of brain injury in Canada is similar to that of Ontario implies that direct total annual costs in Canada for TBI and nTBI treatment in the first year amount to \$331.1 and \$1077.4 million respectively. Citing the Public Health Agency of Canada (PHAC) CIHI reported that the direct cost of head injury in Canada in 2000-2001 was CAD \$151.7 million [16]. This represents approximately $46 \%$ of our estimate of the total cost of TBI in Canada however in their calculation PHAC did not include the cost of rehabilitation and complex continuing care which as we have shown can be substantial.

The present study while rigorous in its methodological approach has certain limitations. Cost calculation was restricted to major health care service categories paid for by the Ontario government. It excluded some government payments for pre-hospital costs diagnostic services, outpatient drugs and supporting equipment and supplies. In this study we took government payer perspective and therefore excluded cost of services paid through private insurance or out-of-pocket. Only direct medical costs were estimated and indirect costs in terms of forgone income of patient or caregiver reduced productivity and disability were unaccounted for. Given that brain injury often leads to lingering health consequences impairments and disability, indirect costs are likely to outweigh the direct ones. Grabow et al. [38] previously assessed indirect costs of head injury through patients' interviews and determined that they accounted for $92 \%$ of the total annual costs while Max and colleagues [39] reported that wages lost due to disability or death represented $88 \%$ of the total cost. Therefore direct medical costs represent only a small fraction of the total burden of ABI. Finally this study included all health system costs and not only those attributable exclusively to ABI. The latter would require eliminating the costs incurred due to health condition other than the primary condition of interest and requires a control group that was not available to us for this study. We also limited our study population to ABI patients who were initially admitted to an acute care hospital and do not include costs of treating patients with mild ABI who are not admitted to hospital since $\mathrm{ABI}$ diagnostic coding in the emergency department and outpatient setting is insufficient to accurately include these patients. We also included all patients with any diagnosis that would include all causes 
of injury including patients who suffered an ABI by any hospital process such as elective surgery. Though we do not have information on the present cohort prior analysis of a similar cohort [36] indicated that $1.8 \%$ of TBI patients and $3.2 \%$ of nTBI patients were not primarily ABI-admissions. This is a small margin of inclusiveness in defining the present cohort. All individuals in the cohort require treatment for $\mathrm{ABI}$ in the health care system. Finally we did not have functional status and were unable to assess the direct costs associated with greater functional impairment directly and rely instead on admission to inpatient rehabilitation to proxy for greater levels of impairment.

Despite the above-mentioned limitations the current study has several important strengths. It provided recent and comprehensive estimate of direct medical costs of ABI across a wide continuum of institutional and community health care settings. This is the first study on direct cost of health care services utilized by patients with nTBI and is only a second study of costs associated with TBI in Canada. Cost estimated in the previous study by Snow et al. [40] were based on patients admitted to a single hospital Sunnybrook Medical Center based in Toronto Ontario and only included hospitalization costs. The use of Ontario administrative healthcare databases permitted us to obtain population-based cost estimates across the entire health care system that are more precise and detailed thus addressing the methodological weaknesses characteristic of other studies $[10,11,20,40,41]$. Finally the longitudinal nature of the study allowed us to examine the evolution of patient costs and utilization patterns over time which could be used for appropriate planning of health care expenses for ABI patients. Future studies however should include cost estimates that capture persons with $\mathrm{ABI}$ who are seen in the emergency room or by physician only as well as the indirect costs. In addition cost analysis could be expanded to statistically analyse what factors contribute most to the economic burden of nTBI and TBI including comorbidities or markers of severity and in comparison to a non-ABI control-group matched on health and functional status. This would be enabled by more fulsome individual data from charts or prospective data collection.

\section{Conclusions}

The main finding of this study is that direct medical costs in the ABI population are substantial with mean cost in the first year post-injury per TBI and nTBI patient being $\$ 32132$ and $\$ 38018$ respectively. Although most expenses occur in the first follow-up year ABI patients continue to use medical services in the second and third year with emphasis shifting from acute care and rehabilitation towards homecare physician services and CCC. Understanding the clinical and health system factors influencing health care utilization in this population and a degree to which health care costs can be controlled is important for planning prevention programs and reducing cost of care among these patients. The data presented here are useful for policy planning and cost-effectiveness analysis.

\section{Competing interests}

The authors declare that they have no competing interests.

\section{Authors' contributions}

AChen and AColantonio conceived and designed the study and acquired the data. DP provided comments and helped to conceive the study. KB and AChen prepared the initial draft of the manuscript. WW and KB interpreted the results and developed the costing methodology. BZ performed the statistical data analysis. All authors critically reviewed read and approved the final manuscript before submission.

\section{Acknowledgements}

This study was supported by funding from the Ontario Neurotrauma Foundation and a grant from the Ministry of Health and Long Term Care to Toronto Rehabilitation Institute as well as funding from the Health System Performance Research Network which is also supported by a grant from the Ontario Ministry of Health and Long-Term Care. We would like to thank the Ministry of Health and Long Term Care in providing us with data and the Canadian Institute for Health Information for advice on data quality and codes. Finally we would like to extend our thanks to Rika Vander Laan for her input in methodology discussions.

\section{Author details}

${ }^{1}$ Toronto Rehabilitation Institute, Toronto, ON, Canada. ${ }^{2}$ Institute of Health Policy Management and Evaluation, University of Toronto, Toronto, ON, Canada. ${ }^{3}$ Institute for Clinical Evaluative Sciences, Toronto, ON, Canada. ${ }^{4}$ Department of Occupational Science and Occupational Therapy, University of Toronto, Toronto, ON, Canada.

Received: 29 August 2011 Accepted: 26 July 2012

Published: 17 August 2012

\section{References}

1. Greenwald BD DM Burnett Miller MA: Congenital and Acquired Brain Injury. Brain Injury: Epidemiology and Pathophysiology. Arch Phys Med Rehabil 2003, 84(3 Suppl 1):S3-7.

2. World Health Organization: Neurological Disorders. Geneva: Public Health Challenges; 2006.

3. ABI Network: Definition of "Acquired Brain Injury". http://www.abinetwork.ca/ downloads/abidefinition.pdf. Accessed: June 152012

4. Centers for Disease Control and Prevention, National Center for Injury Prevention and Control: Traumatic brain injury in the United States: A report to Congress. 1999. [http://www.cdc.gov/traumaticbraininjury/ tbi_report_to_congress.html] Accessed April 52011.

5. Centers for Disease Control and Prevention, National Center for Injury Prevention and Control: Traumatic Brain Injury in the United States: Emergency Departments Visits Hospitalizations and Deaths 2002-2006. 2010. [http://www.cdc.gov/traumaticbraininjury/pdf/blue_book.pdf] Accessed April 52011.

6. Fleminger $S$, Ponsford J: Long term outcome after traumatic brain injury. BMJ 2005, 331:1419-1420.

7. Thornhill S, Teasdale GM, Murray GD, McEwen J, Roy CW, Penny KI: Disability in young people and adults one year after head injury: prospective cohort study. BMJ 2000, 320:1631-1635.

8. Olver JH, Ponsford JL, Curran CA: Outcome following traumatic brain injury: a comparison between 2 and 5 years after injury. Brain Inj 1996, 10(11):841-848.

9. Mazaux JM, Masson F, Levin HS, Alaoui P, Maurette P, Barat M: Long-term neuropsychological outcome and loss of social autonomy after traumatic brain injury. Arch Phys Med Rehabil 1997, 78(12):1316-1320. 
10. Hodgkinson A, Veerabangsa A, Drane D, McCluskey A: Service utilization following traumatic brain injury. J Head Trauma Rehabil 2000, 15(6):1208-1226

11. Vangel SJ, Rapport $L$, Hanks RA, Black KL: Long-term medical care utilization and costs among traumatic brain injury survivors. Am J Phys Med Rehabil 2005, 84:153-160.

12. Phillips VL, Greenspan Al, Stringer AY, Stroble AK, Lehtonen S: Severity of injury and service utilization following traumatic brain injury: The first 3 months. J Head Trauma Rehabil 2004, 19:217-225.

13. Cameron CM, Purdie DM, Kliewer EV, McClure RJ: Ten-year outcomes following traumatic brain injury: A population-based cohort. Brain Inj 2008, 22:437-449.

14. Ghajar J: Traumatic Brain Injury. Lancet 2000, 356:923-929.

15. Canadian Institute of Health Information: NRS length of stay and length of stay efficiency of inpatient rehabilitation clients. http://www.cihi.ca/ClHI-extportal/internet/EN/Quick_Stats/quick+stats/quick_stats_main? $x$ Topic $=$ Hospital\%20Care\&pageNumber $=2 \&$ resultCount $=20 \&$ filterTypeBy $=0$ \& filterTopicBy=5\&autorefresh=1\#. Accessed April 52011.

16. Canadian Institute of Health Information: The Burden of Neurological Diseases, Disorders and Injuries in Canada. Ottawa: $\mathrm{ClHI}$; 2007. https://secure. cihi.ca/free_products/BND_e.pdf. Accessed April 10, 2011.

17. Kraus JF, Nourjah P: The epidemiology of mild uncomplicated brain injury. J Trauma 1988, 28(12):1637-1643.

18. Brener I, Harman JS, Kelleher KJ, Yeates KO: Medical costs of mild to moderate traumatic brain injury in children. J Head Trauma Rehabil 2004, 19(5):405-412

19. Ommaya AK, Ommaya AK, Dannenberg AL, Salazar AM: Causation incidence and costs of traumatic brain injury in the U.S. military medical system. J Trauma 1996, 40(2):211-217.

20. Brooks CA, Lindstrom J, McCray J, Whiteneck GG: Cost of medical care for a population-based sample of persons surviving traumatic brain injury. J Head Trauma Rehabil 1995, 10(4):1-13.

21. Berg J: Economic evidence in trauma: a review. Eur J Health Econom 2004, 5(1):S84-S91.

22. Andlin-Sobocki $P$, Jönsson $B$, Wittchen HU, Olesen J: Cost of disorders of the brain in Europe. Eur J of Neurology 2005, 12(1):1-27.

23. McGarry LJ, Thompson D, Millham FH, Cowell L, Snyder PJ, Lenderking WR, Weinstein MC: Outcomes and costs of acute treatment of traumatic brain injury. J Trauma 2002, 53:1152-1159.

24. Kayani NA, Homan S, Yun S, Zhu BP: Health and economic burden of traumatic brain injury: Missouri 2001-2005. Public Health Rep 2009, 124:551-560.

25. Juurlink D, Preyra C, Croxford R, Chong A, Austin P, Tu J, Laupacis A: Canadian Institute for Health Information Discharge Abstract Database: A validation study. Toronto: Toronto Institute for Clinical Evaluative Sciences; 2006.

26. Canadian Institute of Health Information: National Rehabilitation Reporting System (NRS). http://secure.cihi.ca/cihiweb/en/downloads/ NRS_Update_dec2005_e.pdf. Accessed April 52011.

27. Wodchis WP, Naglie G, Teare GF: Validating Diagnostic Information on the Minimum Data Set in Ontario Hospital-Based Long-Term Care. Medical Care 2008, 46(8):882-887.

28. Ministry of Health and Long-Term Care Health Data Branch. www.mohltcfim. com. Accessed April 52011.

29. Ontario Joint Policy and Planning Committee: Development of rehabilitation patient groups (RPG) case mix classification methodology and weighting system for adult impatient rehabilitation. https://ospace.scholarsportal.info/ bitstream/1873/1253/1/268600.pdf. Accessed April 52011.

30. Colantonio A, Parsons D, Vander Laan R, Zagorski B: Toronto: ABI Dataset Pilot Project Phase 1 Report; Report to the Ontario Neurotrauma Foundation, 2009.

31. Wooder S: Primary Care Compensation Models. Hamilton, Ontario: HNHB Board Presentation; 2011. http://www.hnhblhin.on.ca/uploadedFiles/ Public_Community/Board_of_Directors/Board_Meeting/Primary\%20Care\% 20Compensation\%20Models\%20-\%20LHIN\%20Presentation\%20-\% 20Wooder-FINAL\%20revised.pdf. Accessed November 10, 2011.

32. Collier R: Shift toward capitation in Ontario. CMAJ 2009, 181(10):668-669.

33. Canadian Institute of Health Information: National Health Expenditure Trends 1975 to 2011. Ottawa, Ont: CIHI; 2011. https://secure.cihi.ca/free_products/ nhex_trends_report_2011_en.pdf. Accessed April 10, 2011.
34. Krajden M, Kuo M, Zagorski B, Alvarez M, Yu A, Krahn M: Health care costs associated with hepatitis C: a longitudinal cohort study. Can J Gastroenterol 2010, 24(12):717-726.

35. Krahn MD, Zagorski B, Laporte A, Alibhai SM, Bremner KE, Tomlinson G, Warde $P$, Naglie G: Healthcare costs associated with prostate cancer: estimates from a population-based study. BJU Int 2010, 105(3):338-346.

36. Chen AY, Zagorski B, Parsons D, Vander Laan R, Chan V, Colantonio A: Factors associated with discharge destination from acute care after acquired brain injury in Ontario Canada. BMC Neurol 2012, 12(1):16.

37. Wodchis WP, Camacho X, Dhalla I, Guttman A: Lin E. Anderson G: Targeting Health System Improvement to High Cost Patients. Canadian Association of Health Services and Policy Research. Halifax NS; 2011.

38. Grabow JD, Offord KP, Rieder ME: The cost of head trauma in Olmsted County Minnesota 1970-74. Am J Public Health 1984, 74(7):710-712.

39. Max W, MacKenzie EJ, Rice DP: Head injuries: Costs and consequences. J Head Trauma Rehabil 1991, 6(2):76-91.

40. Snow WG, Macartney-Filgate MS, Schwartz ML, Klonoff PS, Ridgley BA: Demographic and medical characteristics of adult head injuries in a Canadian setting. Can J Surg 1988, 31(3):191-194.

41. Mar J, Arrospide A, Marie J, Larranaga I, Elosegui E, Oliva-Moreno J: The impact of acquired brain damage in terms of epidemiology economics and loss of quality of life. BMC Neurol 2011, 11(1):46.

doi:10.1186/1471-2377-12-76

Cite this article as: Chen et al:: Direct cost associated with acquired brain injury in Ontario. BMC Neurology 2012 12:76.

\section{Submit your next manuscript to BioMed Central and take full advantage of:}

- Convenient online submission

- Thorough peer review

- No space constraints or color figure charges

- Immediate publication on acceptance

- Inclusion in PubMed, CAS, Scopus and Google Scholar

- Research which is freely available for redistribution 